\title{
Evaluation of the BlightPro Decision Support System for Management of Potato Late Blight Using Computer Simulation and Field Validation
}

\author{
Ian M. Small, Laura Joseph, and William E. Fry
}

Department of Plant Pathology and Plant-Microbe Biology, Cornell University, Ithaca, NY 14850.

Accepted for publication 14 August 2015.

\begin{abstract}
Small, I. M., Joseph, L., and Fry, W. E. 2015. Evaluation of the BlightPro decision support system for management of potato late blight using computer simulation and field validation. Phytopathology 105:1545-1554.

The objective of this study was to evaluate the utility of the BlightPro decision support system (DSS) for late blight management using computer simulation and field tests. Three fungicide schedules were evaluated: (i) calendar-based (weekly) applications, (ii) applications according to the DSS, or (iii) no fungicide. Simulation experiments utilized 14 years of weather data from 59 locations in potato-producing states. In situations with unfavorable weather for late blight, the DSS recommended fewer fungicide applications with no loss of disease suppression; and, in

situations of very favorable weather for late blight, the DSS recommended more fungicide applications but with improved disease suppression. Field evaluation was conducted in 2010, 2011, 2012, and 2013. All experiments involved at least two cultivars with different levels of resistance. DSS-guided and weekly scheduled fungicide treatments were successful at protecting against late blight in all field experiments. As expected, DSS-guided schedules were influenced by prevailing weather (observed and forecast) and host resistance and resulted in schedules that maintained or improved disease suppression and average fungicide use efficiency relative to calendar-based applications. The DSS provides an interactive system that helps users maximize the efficiency of their crop protection strategy by enabling well-informed decisions.
\end{abstract}

The late blight disease of potato and tomato, caused by Phytophthora infestans (Mont.) de Bary, is a major concern for producers of these crops. Unexpected epidemics can result in significant crop losses and lead to economic failure for growers for whom potato or tomato crops are the major income source (Fry and Goodwin 1997; Fry et al. 2013). Additionally, the costs of management can be very large. A conservative global estimate of these costs or losses is at least $\$ 6$ billion annually (Haverkort et al. 2008). Although the disease is more problematic in rain-fed agriculture such as in the northeastern United States, sporadically it can also be serious in drier production areas such as the Columbia Basin of central Washington and north-central Oregon regions of the Pacific Northwest (largest production area in the United States) (Fry and Goodwin 1997). For example, the cost of managing a late blight epidemic in the Pacific Northwest in 1995 was estimated at \$30 million (Johnson et al. 1997). On tomato, the disease can be and has been equally devastating. The most recent example occurred in 2009, when infected tomato transplants were distributed via national retail stores who obtained transplants from a national supplier (Fry et al. 2013). The pandemic that followed in the mid-Atlantic and Northeast regions eliminated tomato plants in many organic farms and in many home gardens (Fry et al. 2013).

Management of late blight can be quite complex, involving several factors. These factors include the influence of prevailing weather on the pathogen lifecycle, late blight resistance of the cultivar being grown, fungicide residue on the crop, and pathogen characteristics such as resistance to fungicides. Management tactics involve the use of fungicides and cultural procedures. Cultural procedures are practices designed to reduce the introduction, survival, or infection rate of $P$. infestans. The complexity of the interactions

Corresponding author: I. M. Small; E-mail address: ims56@cornell.edu

*The $\boldsymbol{e}$-Xtra logo stands for "electronic extra" and indicates that two supplementary tables are published online.

http://dx.doi.org/10.1094/PHYTO-05-15-0117-R

(C) 2015 The American Phytopathological Society among these factors makes rational disease management decisionmaking difficult. This complexity creates an opportunity for a decision support system (DSS) to be used to provide science-based information to assist with this decision making.

A DSS can be defined as an interactive computer-based system that helps decision makers utilize data and models to solve unstructured problems under complex and uncertain conditions (Gorry and Morton 1971). DSSs for late blight integrate and organize available information on the pathogen. They may also include the influence of observed and forecasted weather, the effects of cultivar resistance, and the effects of fungicide characteristics and efficacy on the disease. All of these factors are required to make decisions concerning the management of late blight (Cooke et al. 2011).

Successful late blight management requires operational decisionmaking throughout the crop growing season. It is possible to make decisions in the absence of information, although they may be poor decisions. In order to make the best possible decision, it is necessary to be able to both understand and have access to all relevant information (Knight 1997). Due to the significant diversity among growers, communicating relevant information to them and educating them is a challenge. Growers may be conventional or organic, have large or small farms, be independent or part of community-supported agriculture, or be individual home gardeners. Although most growers have access to a plethora of education and extension resources (via internet, e-mail, smart phones, and so on), the information is often scattered or incomplete. A DSS can focus relevant information and tools, curated by experts, to provide a diverse audience with key information to support rational decision making. For example, the association of pathogen phenotypes with particular genotypes and the availability of rapid genotypic analyses enables in-season disease management to be adjusted based on the results of rapid genotypic analyses. This information could be immediately conveyed to growers via a DSS. If such a system had been in place during the 2009 late blight pandemic, important information regarding the sensitivity of the lineages to a highly effective fungicide, mefenoxam, could have been quickly and directly disseminated to growers (Danies et al. 2013). 
Precision agriculture, or "smart farming", aims to optimize the yield per unit of farm land by using the most modern means in a continuously sustainable way, to achieve the best in terms of quality, quantity, and financial return (http://www.beechamresearch.com/ download.aspx?id=40). Technologies such as DSSs are a key component of the smart farming approach. Disease forecasting tools developed in the 20th century were the precursors to plant disease management DSSs (Shtienberg 2013). Forecasters such as BLITECAST (Krause et al. 1975) and Simcast (Fry et al. 1983), FAST (Madden et al. 1978), and the apple scab predictive system (Jones et al. 1980) are examples of tools that were designed to assist farmers with decisions relating to management of potato late blight, early blight, and apple scab, respectively (Shtienberg 2013). Late blight is a disease that has received much attention in terms of disease forecasting (Hardwick 2006). In the United States, several forecasting systems have been developed (Hyre 1954; Krause et al. 1975; Wallin 1962). A common characteristic of these potato late blight forecasts is that they identify a time in the season before which fungicide sprays are needed (Hyre 1954; Wallin 1962). For example, with Blitecast, this interval is identified via 18 severity values (Krause et al. 1975). Following the development of Blitecast, a more comprehensive late blight forecast, Simcast (Fry et al. 1983), which integrates the effects of host resistance and fungicide as well as weather, was developed. Since the 1990s, DSSs have been developed in many countries to assist with the management of plant diseases such as potato late blight, apple scab, cereal leaf diseases, and grape downy mildew (Shtienberg 2013). In Europe, several DSSs for late blight have been developed using various disease forecasting systems and models. A list of these DSSs can be found on the Euroblight website (a potato late blight network for Europe) (http://euroblight.net/control-strategies/dssoverview/). In North America, the BlightPro DSS was developed to integrate the effects of weather, host resistance and fungicide on disease progress in order to improve in-season late blight management. The BlightPro DSS has been available through several sources, including via www.USAblight.org. The BlightPro DSS (http://blight.eas.cornell.edu/blight/) is an internet-based platform and is made up of several components. Growers identify the location of their production unit (latitude and longitude of field) and the system automatically obtains observed weather data from the nearest available weather station, and location-specific $\left(2.5-\mathrm{km}^{2}\right.$ grid $)$ forecast weather data from the National Weather Service-National Digital Forecast Database. These weather data along with crop and management information are used to drive disease forecasting tools Blitecast and Simcast (Fry et al. 1983; Krause et al. 1975), as well as a mechanistic late blight disease simulator, LATEBLIGHT 2004 (LB2004) (Andrade-Piedra et al. 2005b). To inform their decisionmaking process, decision-makers can utilize the cultivar late blight resistance database on the system, use the forecast information from Blitecast or Simcast, run in-season simulations with LB2004, and obtain up-to-date information about the sensitivity of pathogen lineages to mefenoxam. In addition, the system includes an integrated alert system, which enables the user to receive notifications about upcoming critical thresholds for intervention (fungicide application) via e-mail or text message. For a comprehensive description of the development and implementation of BlightPro, see the work of Small et al. (2015). The objective of this study was to evaluate the utility of the BlightPro DSS using computer simulation experiments and field experiments. Utility was defined as the ability of the DSS to enable the suppression of late blight while increasing the efficiency of fungicide use.

\section{MATERIALS AND METHODS}

Evaluation by computer simulation. Simulation analyses were carried out following the approach used by Shteinberg and associates (Shtienberg and Fry 1990; Shtienberg et al. 1989). The LB2004 disease model integrated with fungicide submodels (Andrade-Piedra et al. 2005b) was used to evaluate fungicide scheduling methods. The LB2004 model describes pathogen development as a function of weather, fungicide, and host resistance; fungicide dynamics are described as a function of weather and time since the last application. This disease model was developed and validated in small field plots (Andrade-Piedra et al. 2005a).

Simulation experiments used 14 years of meteorological data (2000 to 2013) recorded from locations in Maine, Massachusetts, New York, North Carolina, North Dakota, and Wisconsin. Weather data were obtained from the Northeast Regional Climate Center. Only locations and years for which there was less than $2 \%$ missing weather data between the date of emergence and vine kill were used. This criterion resulted in 768 environments with suitable weather data. The following common parameters were used for each season: the length of the season (from the date of planting to vine kill) was 110 days; median emergence occurred on the 18th day after planting; and the initial level of late blight was $0.001 \%$ disease severity (one lesion per 10 plants). The protectant fungicide chlorothalonil was applied at a rate of $1.34 \mathrm{~kg}$ active ingredient/ha. Simulations were conducted using susceptible, moderately susceptible, and moderately resistant cultivars for each disease scenario.

Two scenarios for initial appearance of disease were investigated for each location in each season. First, the initial appearance of late blight was set to occur 6 days after the accumulation of 18 Blitecast severity values, because this was found to be the observed mean time of late blight appearance in field experiments where inoculated potato tubers were planted (Doster et al. 1989; Shtienberg and Fry 1990). Second, the initial appearance of late blight was a random date between 18 Blitecast severity values and the end of the season; this scenario was included to represent the variability in initial late blight occurrence due to differences in inoculum source. We have limited our study to environments with temperate climates in which the cold winter eliminates susceptible host plants between growing seasons, requiring the disease to be initiated each growing season. The use of 18 Blitecast severity values to predict initiation of disease is appropriate in such climates. In environments where there is susceptible host tissue available year-round, it is possible that the disease might be initiated at any time after emergence. This simulation study does not address the scenario where there is susceptible host tissue available year-round.

The efficacy of three spray-scheduling methods in suppressing late blight were evaluated.

(i) Calendar-based strategy. Weekly sprays were initiated 35 days after planting and continued until the end of the season.

(ii) DSS strategy. The DSS was used to obtain location-specific spray recommendations based on forecast programs Blitecast and Simcast, which are integrated within the DSS. Sprays were initiated when 18 Blitecast severity values had accumulated since median emergence. Subsequent applications were timed according to the effect of weather on the pathogen (accumulation of blight units) and on fungicide weathering (accumulation of fungicide units), as obtained from Simcast reports within the DSS (Fry et al. 1983). Although DSS users could potentially also utilize information from the LB2004 model when deciding whether or not to spray, the model was not used to schedule sprays for the simulation analyses. This was avoided because the same model was utilized to conduct the simulation analyses.

(iii) Unsprayed. No fungicides were applied throughout the season.

Schedules for fungicide applications were determined separately for each spray-scheduling method and were then simulated with the pathogen model. In total, 2,478 different simulations (59 locations $\times$ 14 years $\times$ three susceptibility groups) were conducted for each of the following spray-scheduling methods: (i) conventional, (ii) DSS strategy, and (iii) unsprayed.

After elimination of environments with missing weather, spray scheduling by the DSS was compared with calendar-based approaches to fungicide scheduling in 6,912 simulations (equivalent to 768 field experiments). Comparisons were made for (i) the number of fungicide applications scheduled, (ii) area under the disease progress curve 
(AUDPC), (iii) disease suppression relative to the unsprayed control, and (iv) efficiency of fungicide use. Fungicide use efficiency $(E)$ was defined as the percent disease control per application and was calculated following the approach used by Shtienberg and Fry (1990). Equation 1 is corrected from that described in 1990 as per personal correspondence with the author:

$$
\text { Fungicide use efficiency }(E)=\{[(A u-A m) / A u] / N\} 100
$$

in which $A m=$ simulated AUDPC for a spray scheduling method, $A u=$ simulated AUDPC for untreated crop, and $N=$ number of applications scheduled by a method.

Field validation. The BlightPro DSS was evaluated in field trials over 4 years, on susceptible 'Yukon Gold' (in 2011, 2012, and 2013), moderately susceptible 'Katahdin' (in 2010), and moderately resistant 'Kennebec' (in 2010 to 2012) potato cultivars at the Homer C. Thompson Vegetable Research Farm, Freeville, NY. All experiments involved at least two cultivars with different levels of resistance. Potato seed pieces were planted on 6 July 2010, 30 June 2011, 9 July 2012, and 8 July 2013. Field experiments were planted at a later date than would be typical for the area, to ensure healthy foliage late in the season. This was done to allow inoculation at a later date in the season to avoid generating a source of inoculum for growers in the area. The experimental design was a randomized complete block design with four blocks. Plots were six rows, $3.68 \mathrm{~m}$ long, with 0.86-m spacing between rows and 0.23-m (2010 and 2011) or 0.30-m (2012, 2013) seed piece spacing. The resultant plot size was 3.68 by $4.3 \mathrm{~m}$. The soil type was a Howard gravelly loam. Fertilization was 13-13-13 (N-P$\mathrm{K})$ at $1,290 \mathrm{~kg} \mathrm{ha}^{-1}$ banded in the row at planting. Fungicide treatment programs were compared with an untreated control for each potato cultivar (Table 1). Two fungicides were utilized in the experiments: chlorothalonil, $720 \mathrm{~g} \mathrm{liter}^{-1}$ (Bravo WS), at a rate of 1.75 liters ha ${ }^{-1}$ (2010 to 2013); and mefenoxam, $39.5 \mathrm{~g}$ liter $^{-1}$, in combination with chlorothalonil, $400 \mathrm{~g}$ liter-1 $^{-1}$ (Ridomil Gold Bravo), at a rate of 2.92 liters ha-1 (2012). Fungicide treatments were applied based on either a calendar- or DSS-based schedule. Fungicide treatments were applied with an Air Tec side boom sprayer. The Air Tec sprayer is an air-assist sprayer which improves coverage by decreasing droplet size, while also increasing penetration into dense plant canopies. The sprayer output was 187 liters ha ${ }^{-1}$ at $40 \mathrm{psi}$, using a diaphragm pump. Sprayer speed was $4.83 \mathrm{~km} \mathrm{~h}^{-1}$. The boom was $6.70 \mathrm{~m}$ long, with nozzles spaced $0.41 \mathrm{~m}$ apart. Hollow-cone nozzles (TX-VS12), with low pressure check valves to eliminate drip, were used.

In all field experiments, weather conditions were conducive for late blight, with 18 Blitecast severity values being accumulated on 4 August 2010, 7 August 2011, 30 July 2012, and 14 June 2013. Sprays for the DSS schedule were initiated using chlorothalonil when the Blitecast severity values had accumulated to 18 and plants had reached at least 15 to $20 \mathrm{~cm}$ in height. Due to the late planting date, 18 severity values were reached before plants were 15 to $20 \mathrm{~cm}$

TABLE 1. Field evaluation of fungicide scheduling strategies on potato cultivars with different levels of resistance to late blight

\begin{tabular}{|c|c|c|c|c|c|c|}
\hline Year, cultivar & Blight $^{v}$ & Schedule $^{\mathrm{w}}$ & Fungicide & $N^{\mathrm{x}}$ & AUDPC ${ }^{y}$ & $\mathrm{E}(\%)^{\mathrm{z}}$ \\
\hline \multicolumn{7}{|l|}{2010} \\
\hline \multirow[t]{2}{*}{ Katahdin } & MS & DSS & $\mathrm{C}$ & 6 & $0.02 \mathrm{a}$ & 16.7 \\
\hline & & Calendar-based & $\mathrm{C}$ & 8 & $0.02 \mathrm{a}$ & 12.5 \\
\hline \multirow[t]{3}{*}{ Kennebec } & MR & DSS & $\mathrm{C}$ & 5 & $0.02 \mathrm{a}$ & 20.0 \\
\hline & & Calendar-based & $\mathrm{C}$ & 8 & $0.26 \mathrm{a}$ & 12.3 \\
\hline & & Unsprayed & None & 0 & $36.1 \mathrm{~b}$ & \\
\hline \multirow{2}{*}{ Yukon Gold } & & Calendar-based & $\mathrm{C}$ & 8 & $0.10 \mathrm{a}$ & 12.5 \\
\hline & & Unsprayed & None & 0 & $821.4 \mathrm{c}$ & \\
\hline \multirow[t]{3}{*}{ Kennebec } & MR & DSS & $\mathrm{C}$ & 5 & $0.63 \mathrm{a}$ & 20.0 \\
\hline & & Calendar-based & $\mathrm{C}$ & 8 & $0.14 \mathrm{a}$ & 12.5 \\
\hline & & Unsprayed & None & 0 & $504.9 \mathrm{~b}$ & \\
\hline \multicolumn{7}{|l|}{2012} \\
\hline \multirow{2}{*}{ Yukon Gold } & & & $\mathrm{C}$ & 3 & & \\
\hline & & Unsprayed & None & 0 & $174.3 \mathrm{~b}$ & \\
\hline \multirow[t]{7}{*}{ Kennebec } & MR & DSS & $\mathrm{C}$ & 3 & $0.01 \mathrm{a}$ & 33.3 \\
\hline & & Calendar-based & $\mathrm{C}$ & 6 & $0.00 \mathrm{a}$ & 16.7 \\
\hline & & DSS & $\mathrm{M}+\mathrm{C}$ & 2 & $0.00 \mathrm{a}$ & 25.0 \\
\hline & & & $\mathrm{C}$ & 2 & & \\
\hline & & Calendar-based & $M+C$ & 3 & $0.01 \mathrm{a}$ & 16.7 \\
\hline & & & $\mathrm{C}$ & 3 & & \\
\hline & & Unsprayed & None & 0 & $170.3 \mathrm{~b}$ & \\
\hline \multicolumn{7}{|l|}{2013} \\
\hline \multirow[t]{2}{*}{ Yukon Gold } & $\mathrm{S}$ & DSS & $\mathrm{C}$ & 5 & $0.00 \mathrm{a}$ & 20.0 \\
\hline & & Calendar-based & $\mathrm{C}$ & 5 & $0.00 \mathrm{a}$ & 20.0 \\
\hline
\end{tabular}

${ }^{\mathrm{v}}$ Late blight resistance of the cultivar: $\mathrm{S}=$ susceptible, $\mathrm{MS}=$ moderately susceptible, and MR $=$ moderately resistant decision support system.

${ }^{\mathrm{w}}$ Schedule: method used to schedule fungicide applications. DSS = Simcast schedule for chlorothalonil $(\mathrm{C})$ or mefenoxam + chlorothalonil $(\mathrm{M}+\mathrm{C}) ; \mathrm{Calendar}=$ 7-day interval for $\mathrm{C}$ or 14-day interval for $\mathrm{M}+\mathrm{C}$. Based on label recommendations, chlorothalonil was applied 7 days after each $\mathrm{M}+\mathrm{C}$ application for the calendar schedule, or when Simcast fungicide unit threshold was reached for DSS schedule.

$x$ Number of sprays.

y AUDPC $=$ area under the disease progress curve. All values are the mean of four replicates. Means followed by the same letter within each experiment (year) are not significantly different according to Tukey Kramer honestly significant difference $(P<0.05)$.

${ }^{\mathrm{z}}$ Fungicide use efficiency (E) refers to the average percent disease control achieved per fungicide application. 
in height for all experimental years; therefore, sprays were delayed until plants were approximately 15 to $20 \mathrm{~cm}$ tall. Sprays for the conventional treatment were initiated using chlorothalonil when plants were approximately 15 to $20 \mathrm{~cm}$ in height. In the 2012 field experiment, treatments including systemic fungicide (mefenoxam + chlorothalonil) were incorporated. The change from contact (chlorothalonil) to systemic fungicide sprays in the 2012 experiment was initiated for both the DSS treatment and the conventional treatment when late blight lesions were first observed in the nearby untreated experimental plots (inoculum source).

The DSS evaluation field experiments relied on natural infections from infected plants in experimental plots (artificially inoculated) at a distance of 0.5 to $0.8 \mathrm{~km}$ in each year. Genotypes of $P$. infestans identified in DSS evaluation experiments were determined by microsatellite analysis using an established protocol (Lees et al. 2006). Disease ratings were determined by visually assessing each plot for the percentage of diseased foliage caused by late blight using a method described by Fry (1977). Disease severity was rated every 3 to 10 days, with more frequent assessments occurring during rapid epidemic development.

Statistical analyses. Simulation analyses. Spray-scheduling methods were compared based on the number of fungicide applications scheduled, AUDPC, disease suppression, and efficiency of fungicide use. Fungicide treatment, scheduled by either the DSS or calendar based, significantly suppressed disease relative to the unsprayed treatment (see Results). For this reason, the focus of the statistical analysis was to investigate differences between DSS and calendar-based fungicide schedules for their disease suppression.

To compare the efficacy of DSS-recommended treatment schedules with calendar-based treatment schedules, the disease suppression due to the treatment was calculated. Disease suppression (T) was defined as follows: the reduction in AUDPC due to the treatment $\left(\mathrm{AUDPC}_{\text {Unsprayed }}-\mathrm{AUDPC}_{\text {Treatment }}\right)$, converted to a proportion of the unsprayed control at that location and for the appropriate year. This proportion $(\mathrm{T})$ is the reduction in disease due to the treatment (disease suppression) in equation 2.

$$
\text { Disease suppression }(T)=\frac{A U D P C_{\text {Unsprayed }}-A U D P C_{\text {Treatment }}}{A U D P C_{\text {Unsprayed }}}
$$

A general linear model was used (JMP Pro, version 11.2.0; SAS Institute Inc., Cary, NC), where the response was logittransformed disease suppression (T). The transformation was conducted to satisfy statistical assumptions for the statistical model. Environments (year $x$ at location $y$ ) where simulated disease severity (AUDPC) for the unsprayed treatment $<100$ were excluded, which retained 673 environments. We set this minimum threshold for disease in the untreated plots to ensure that the two fungicide treatment schedules were compared for their disease suppression in environments in which there was at least some disease. Fungicide treatment schedules, cultivar late blight resistance, location year, and the interaction between fungicide treatment schedule and cultivar resistance were considered fixed effects. Least squares means (LS means) were compared using a Tukey honestly significant difference (HSD) posthoc test $(P=0.05)$. Results were presented as percent disease suppression by back transforming LS means to proportions and then multiplying by 100 to obtain a percentage. We used the result for a weekly schedule on susceptible cultivars as a baseline for adequate disease suppression. This is because we know that a weekly schedule on more resistant cultivars obviously increases disease suppression but is also likely to use excessive fungicide.

To investigate the fungicide use efficiency of different treatments, a nonparametric test was conducted on the means for each combination of treatment and cultivar resistance (e.g., DSS schedule on susceptible cultivar). A nonparametric test was conducted because assumptions of normality were not satisfied. The response was fungicide use efficiency (E). A nonparametric comparison for each pair of treatment means was conducted using the Wilcoxon each pair test (Hsu 1996).

Field experiments. Spray-scheduling methods (treatments) were compared based on the number of fungicide applications scheduled, mean AUDPC, and fungicide use efficiency (E) for each treatment. A general linear mixed model was used (JMP Pro, version 11.2.0; SAS Institute Inc.), where the response was arcsine square root transformed AUDPC. Fungicide treatment schedule, cultivar late blight resistance, and the interaction between fungicide treatment schedule and cultivar resistance were considered fixed effects. The effect of block in each experiment was treated as a random effect. Each experiment (year) was analyzed separately. The restricted maximum likelihood method was used for the mixed model. Least squares means were compared using a Tukey HSD posthoc test $(P=0.05)$.

\section{RESULTS}

Comparison of spray-scheduling methods via computer simulation. Number of fungicide applications scheduled. In some cases, the DSS recommended more fungicide applications and, in other cases, the DSS recommended fewer applications than the calendar-based schedule (Fig. 1; Supplementary Table S1). For susceptible cultivars, the DSS recommended $24 \%$ more fungicide applications (median of 14 sprays) than the weekly schedule (11 sprays). The range was -91 to $+91 \%$. For moderately susceptible cultivars, the DSS recommended $15 \%$ fewer applications (median of 10 sprays) relative to a 7 -day schedule (11 sprays). The range was -91 to $+36 \%$. For moderately resistant cultivars, the DSS recommended a reduction in fungicide application of $36 \%$ (median of seven sprays) relative to a 7-day schedule (11 sprays). The range was -91 to $0 \%$. In addition to the effect of cultivar resistance on numbers of recommended sprays, the favorability of prevailing weather for late blight influenced the number of recommended applications (Fig. 2). Higher numbers of applications were recommended when conditions were favorable for disease development. In the DSS-treated experiments, the median spray intervals were 6 days between sprays for susceptible cultivars, 9 days for moderately susceptible cultivars, and 12 days for moderately resistant cultivars (Fig. 1).

Experiments when disease was initiated after the accumulation of 18 severity values (scenario 1). $A U P D C$. The wide range of weather conditions in the 768 individual simulation experiments had a large effect on the simulated severity of late blight. Due to the nonnormal distribution of the raw AUDPC data, medians and interquartile ranges (IQR) are presented as descriptive statistics (Fig. 3). The yearly median AUDPC for the unsprayed control ranged from 4,038 in 2012 to 5,912 in 2003. The median AUDPC for the unsprayed strategy was 5,253 (IQR = 1,968) for susceptible, 4,898 (IQR = 2,092) for moderately susceptible, and 2,683 (IQR $=2,934$ ) for moderately resistant cultivars. As expected, fungicide applications reduced these numbers dramatically. The median AUDPC for the DSS strategy was 49 (IQR = 379) for the susceptible, 569 (IQR = 1439) for the moderately susceptible, and $22.5(\mathrm{IQR}=146)$ for the moderately resistant cultivars, respectively. This can be compared with the average AUDPC values for the calendar-based strategy of $1,729(\mathrm{IQR}=3,326)$ for the susceptible, 760 (IQR = 2,242) for the moderately susceptible, and 4.2 (IQR $=29$ ) for the moderately resistant cultivars, respectively.

Fungicide treatment, either DSS or calendar based, dramatically reduced disease relative to the unsprayed treatment. This was supported by the fact that the $95 \%$ confidence interval around the mean of the unsprayed treatment for each resistance category did not overlap with the $95 \%$ confidence interval around the mean of either of the fungicide schedules. For this reason, the statistical analysis was focused on the difference between fungicide schedules.

Disease suppression. The disease suppression for the DSS treatment for each category of resistance was compared with a baseline of disease suppression achieved with weekly sprays on 
a susceptible cultivar. The DSS-recommended spray schedules suppressed late blight to lower levels on susceptible cultivars (99.7\% disease suppression) than did the weekly schedule on susceptible cultivars $(98.6 \%$ disease suppression $)(P<0.0001)$. The DSS-recommended schedule for moderately susceptible cultivars ( $97.7 \%$ disease suppression) differed significantly from the calendarbased schedule for susceptible cultivars (98.6\% disease suppression) $(P<0.0001)$. No significant difference in disease suppression was observed between the DSS strategy for the moderately resistant cultivars ( $99.1 \%$ disease suppression) and the calendarbased schedule for susceptible cultivars $(98.6 \%$ disease suppression) $(P=0.12)$.

Fungicide use efficiency. For all cultivar resistance categories, sprays scheduled according to the DSS strategy had a significantly higher average fungicide use efficiency $(E)(P<0.0001)$ than the calendar-based strategy (Fig. 4). For susceptible cultivars, average disease control per application was $5.7 \%$ for the calendar-based schedule and $7.7 \%$ for the DSS-recommended schedule $(35 \%$ difference in fungicide use efficiency). For moderately susceptible cultivars, average disease control per application was $6.8 \%$ for the calendar-based schedule and $9.7 \%$ for the DSS-recommended schedule ( $43 \%$ difference in fungicide use efficiency). For moderately resistant cultivars, average disease control per application was $8.8 \%$ for the calendar-based schedule and $14.2 \%$ for the DSS-recommended schedule (61\% difference in fungicide use efficiency).

Experiments with disease initiation at a random date after accumulation of 18 severity values (scenario 2). Obviously, the fungicide application scheduling was independent of the initiation of the disease in the simulation experiments; therefore, the fungicide schedules for the random initiation scenario were the same as the schedules for the "6 days after 18 severity values" scenario.

$A U D P C$. When the appearance of disease in simulations was set to occur at a random date after the accumulation of 18 severity values, instead of being fixed at 6 days after accumulation of 18 severity value, the AUDPC was reduced for all treatments $(>60 \%$ reduction in average AUDPC) (Fig. 5). The median AUDPC values for the unsprayed strategy were 1,065 (IQR $=3,428$ ) for susceptible, $689(\mathrm{IQR}=2,998)$ for moderately susceptible, and $17(\mathrm{IQR}=1,169)$ for moderately resistant cultivars (Supplementary Table S2). The median AUDPC values for the DSS strategy were 0.5 (IQR = 17) for susceptible, $1.8(\mathrm{IQR}=115)$ for moderately susceptible, and 0.3 $(\mathrm{IQR}=6)$ for moderately resistant cultivars. The median AUDPC values for the calendar-based strategy were $0.9(\mathrm{IQR}=120)$ for susceptible, $0.4(\mathrm{IQR}=20)$ for moderately susceptible, and 0.1 $(\mathrm{IQR}=0.4)$ for moderately resistant cultivars.

Disease suppression. Again, fungicide applications effectively reduced disease severity for all resistance categories. The DSSrecommended spray schedules suppressed late blight to lower levels on susceptible cultivars (99.8\% disease suppression) than did the weekly schedule ( $98.8 \%$ disease suppression) $(P<0.0001)$. However, the DSS-recommended schedule for moderately susceptible cultivars (98.4\% disease suppression) resulted in higher disease levels than did the calendar-based schedule for susceptible cultivars (98.8\% disease suppression) $(P<0.0001)$. For moderately resistant cultivars, the DSS-recommended spray schedules suppressed
A

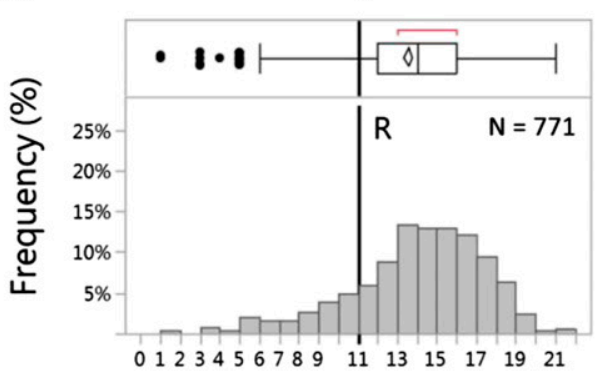

B

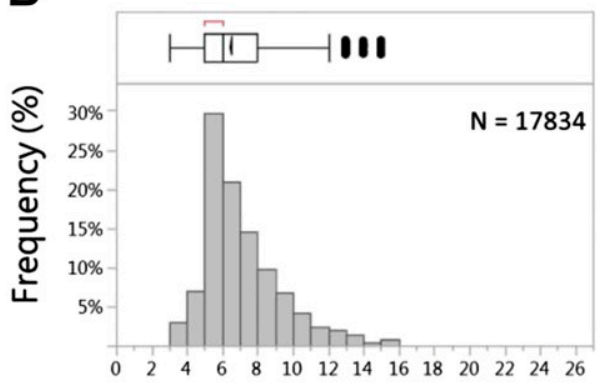

Moderately Susceptible

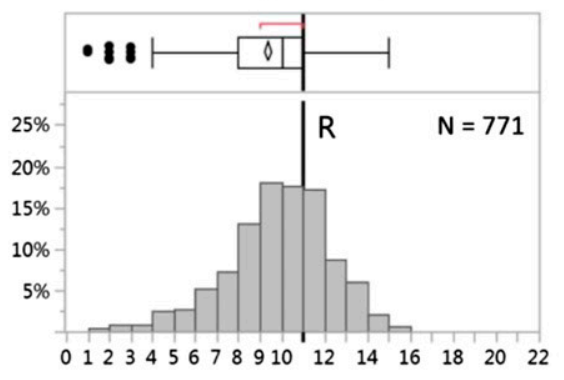

No. of sprays scheduled by the DSS

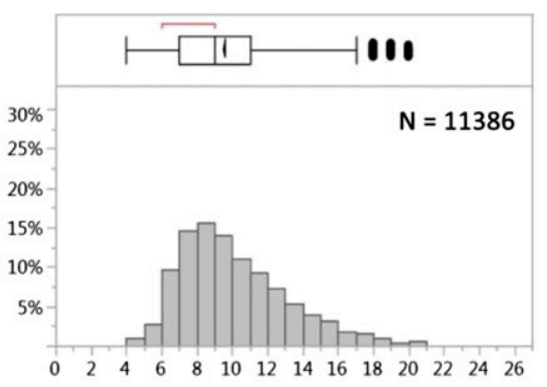

Moderately Resistant

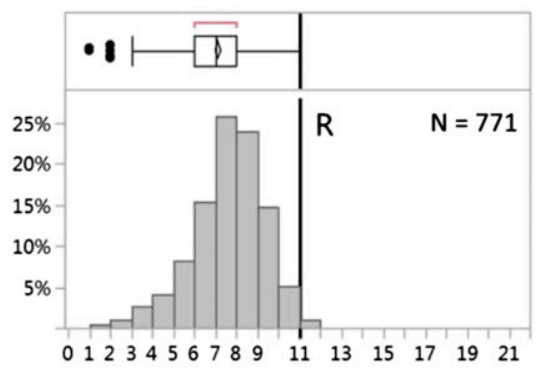

\section{Application interval (days)}

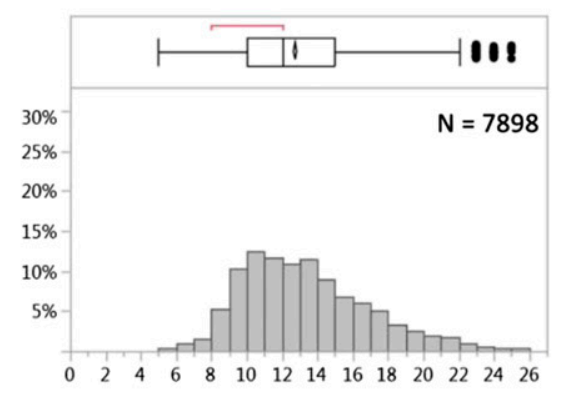

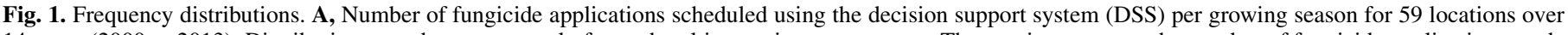

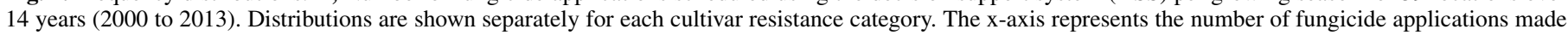

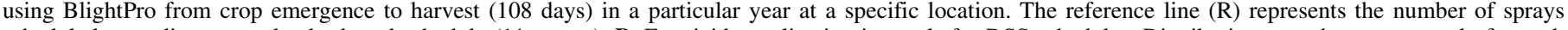

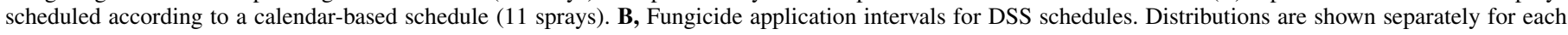

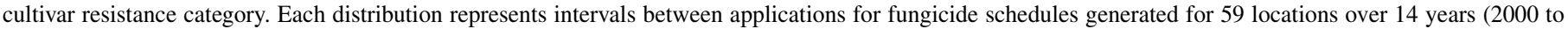

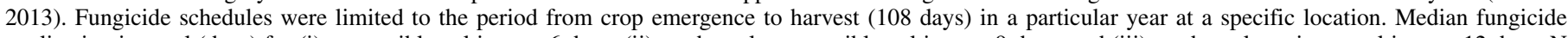

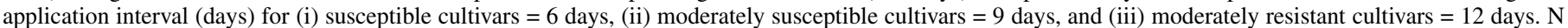

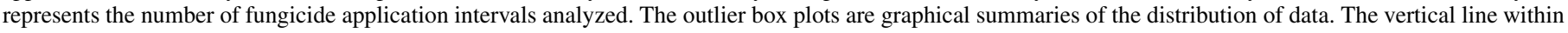

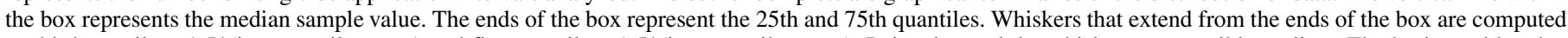

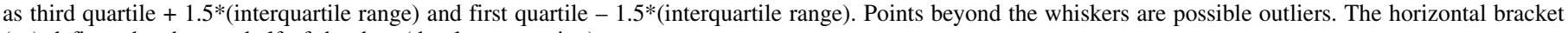
( $\neg$ ) defines the shortest half of the data (the densest region). 
late blight to lower levels (99.6\% disease suppression) than did the weekly schedule on susceptible cultivars $(98.8 \%$ disease suppression) $(P<0.0001)$.

Fungicide use efficiency. Under the random inoculation date scenario, fungicide use efficiency for the DSS-recommended schedule was higher for the moderately susceptible and moderately resistant cultivars, but not susceptible cultivars, relative to the calendar-based schedule (Fig. 4). For susceptible cultivars, average disease control per application was $7.8 \%$ for the calendar-based schedule and $7.3 \%$ for the DSS-recommended schedule (7\% difference in fungicide use efficiency). For moderately susceptible cultivars, average disease control per application was $8.3 \%$ for the calendar-based schedule and $9.7 \%$ for the DSS-recommended schedule (17\% difference in fungicide use efficiency). For moderately

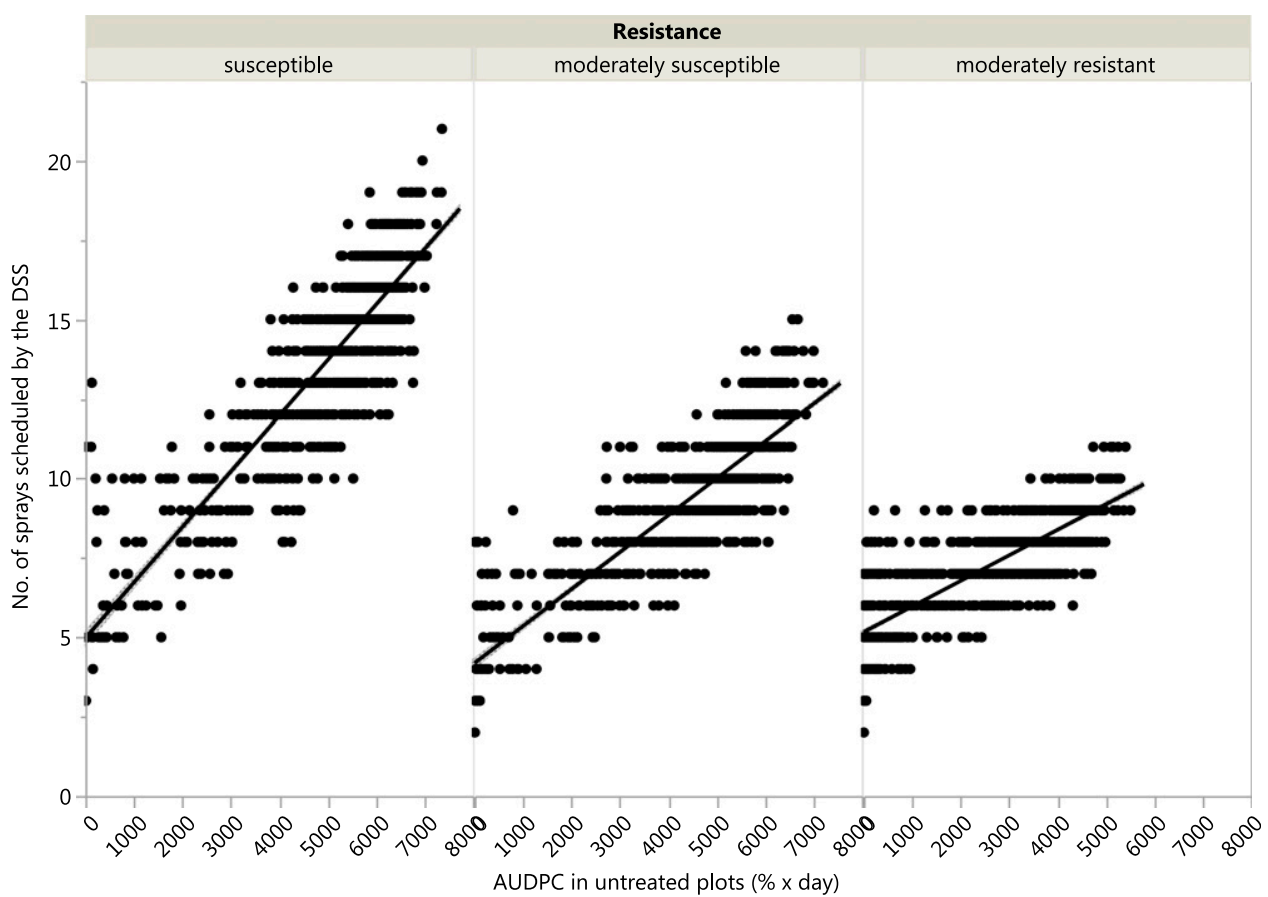

Fig. 2. Illustration of the responsiveness or sensitivity of the decision support system (DSS) to the suitability of weather conditions for Phytophthora infestans. The suitability of weather conditions to P. infestans was estimated by the intensity of simulated epidemics in untreated plots and the sensitivity of the DSS by the number of scheduled sprays. In simulations, the epidemic was initiated 6 days after 18 severity values had accumulated. Each point represents the final area under the disease progress curve (AUDPC) in untreated plots for 1 year at a particular location. The line in each panel represents the best fit line to the data and is simply presented to highlight the relationship between number of sprays scheduled by the DSS and the AUDPC in untreated plots.

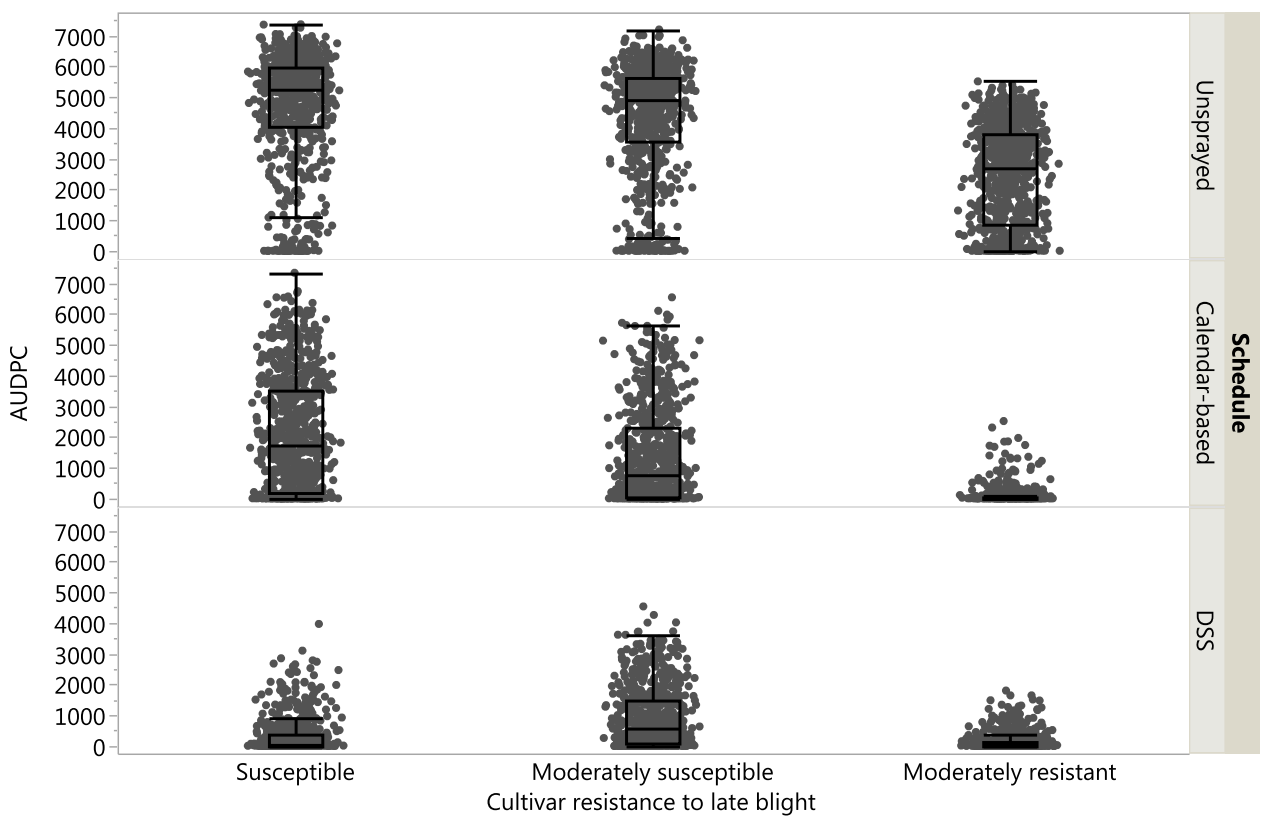

Fig. 3. Summary of simulated disease severity (area under the disease progress curve [AUDPC]) for 59 locations over 14 years (2000 to 2013) (scenario 1). Three fungicide scheduling methods were evaluated for their ability to suppress late blight (calendar-based schedule, decision support system [DSS]-informed schedule, and unsprayed). Each scheduling method was evaluated for three categories of cultivar resistance to late blight. Late blight was simulated to occur 6 days after 18 Blitecast severity values had accumulated. Each point represents the final AUDPC for a schedule in 1 year at a particular location. Box plots overlaying the points are a graphical summary of the distribution of data. The horizontal line within the box represents the median sample value. The ends of the box represent the 25 th and 75th quantiles. Whiskers that extend from the ends of the box are computed as third quartile $+1.5^{*}$ (interquartile range) and first quartile $-1.5^{*}($ interquartile range). 
resistant cultivars, average disease control per application was $9.0 \%$ for the calendar-based schedule and $12.8 \%$ for the DSSrecommended schedule ( $42 \%$ difference in fungicide use efficiency).

Field validation. Late blight was first observed in the unsprayed plots on 15 September 2010, 12 September 2011, 2 September 2012, and 1 September 2013. In all of these experiments, favorable weather conditions for late blight resulted in rapid development of the disease in the untreated plots. In all experiments, both fungicide treatments (DSS strategy or calendar-based schedule) significantly $(P<0.05)$ suppressed late blight (Table 1). No significant difference in disease suppression was observed between the DSS strategy and the calendar-based schedule for all three categories of cultivar resistance $(P<0.05)$.
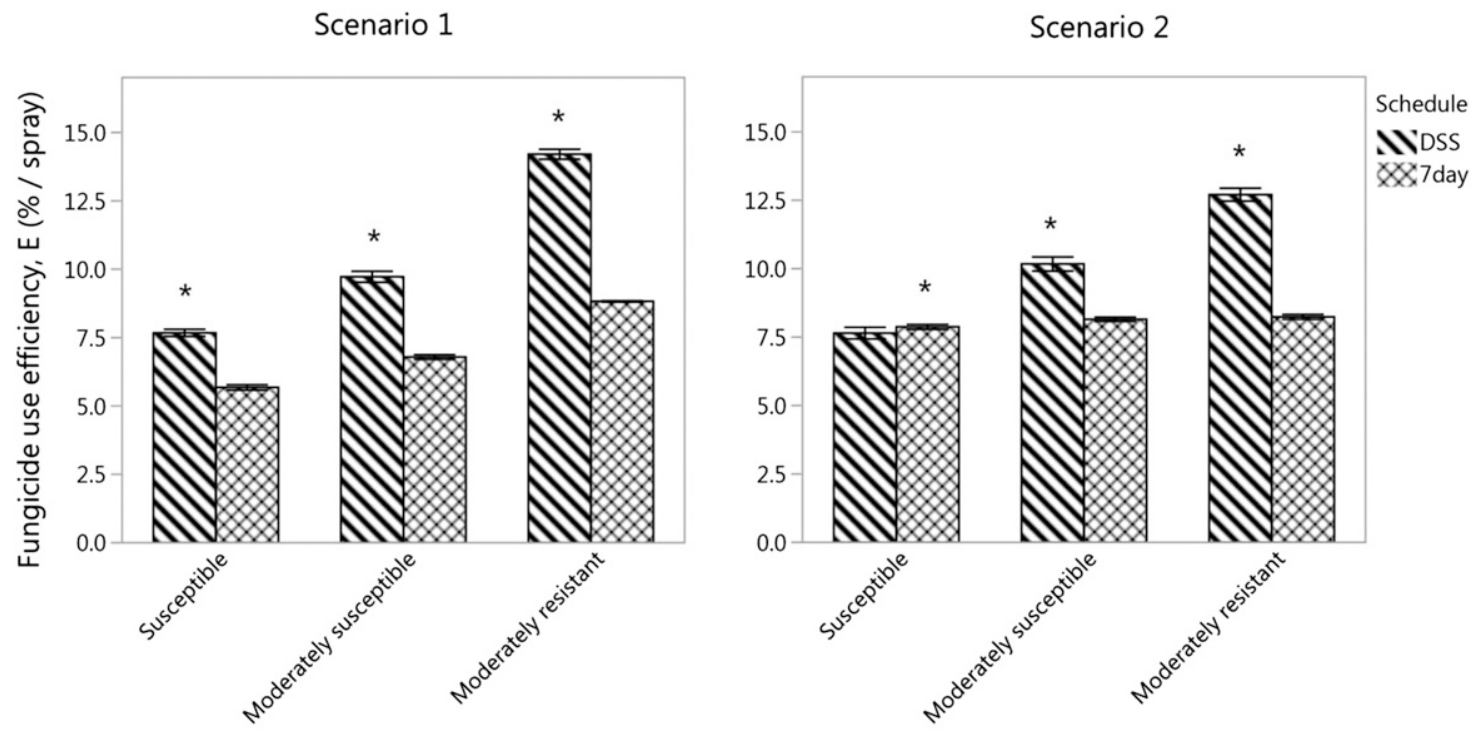

Cultivar resistance to late blight

Fig. 4. Fungicide use efficiency (reduction in area under the disease progress curve per spray) of decision support system (DSS) strategy and calendar-based (7-day) strategy, under two disease initiation scenarios. Scenario 1: late blight was simulated to occur 6 days after 18 Blitecast severity values had accumulated. Scenario 2: late blight was simulated to occur at a random date between 6 days after 18 Blitecast severity values had accumulated and the end of the season. Fungicide use efficiency was calculated for both strategies on three categories of cultivar resistance to late blight. Each error bar is constructed using 1 standard error from the mean. An asterisk indicates statistically significant difference in efficiency between DSS and calendar-based strategy $(P \leq 0.05)$ within a resistance category. Statistics are based on nonparametric comparison for each pair of treatment means conducted using a Wilcoxon each-pair test (Hsu 1996).

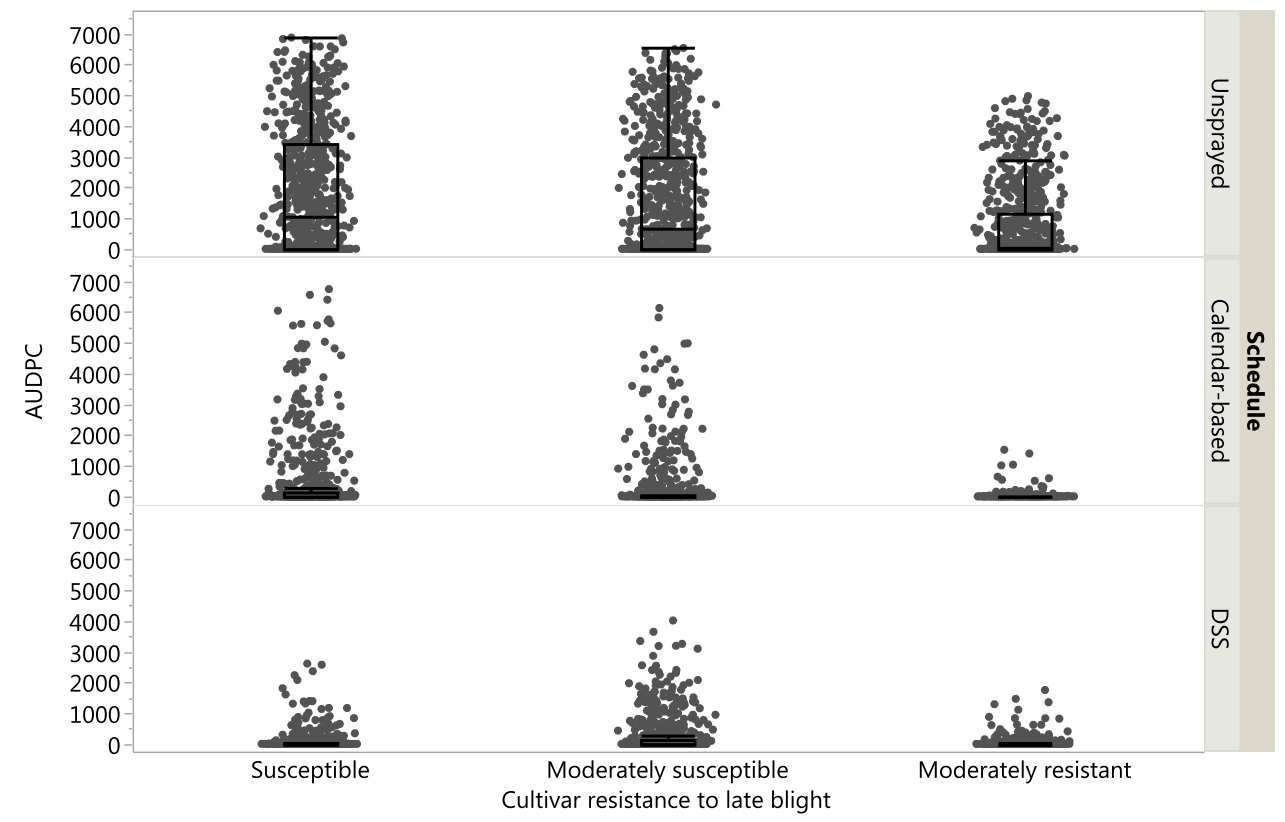

Fig. 5. Summary of simulated disease severity (area under the disease progress curve [AUDPC]) for 59 locations over 14 years (2000 to 2013 ) (scenario 2). Three fungicide scheduling methods were evaluated for their ability to suppress late blight (calendar-based schedule, decision support system [DSS]-informed schedule, and unsprayed). Each scheduling method was evaluated for three categories of cultivar resistance to late blight. Late blight was simulated to occur at a random date between 6 days after 18 Blitecast severity values had accumulated and the end of the season. Each point represents the final AUDPC for a schedule in 1 year at a particular location. Box plots overlaying the points are a graphical summary of the distribution of data. The horizontal line within the box represents the median sample value. The ends of the box represent the 25th and 75th quantiles. Whiskers that extend from the ends of the box are computed as third quartile + $1.5 *$ (interquartile range) and first quartile $-1.5 *$ (interquartile range). 
In experiments where chlorothalonil alone was used on a susceptible cultivar (Yukon Gold), the DSS strategy scheduled the same number (2012 and 2013 experiments) or slightly more applications (one additional spray in 2011 experiment) than did the calendar-based approach (Table 1). For the moderately susceptible cultivar (Katahdin), the DSS strategy scheduled fewer applications (six sprays) when compared with the calendar-based approach (eight sprays) (Table 1). For the moderately resistant cultivar (Kennebec), the DSS strategy scheduled fewer applications (five, five, and three sprays in 2010, 2011, and 2012, respectively) when compared with the calendar-based approach (eight, eight, and six sprays in 2010, 2011, and 2012, respectively) (Table 1).

In the 2012 experiment, a fungicide mixture (mefenoxam + chlorothalonil) alternated with chlorothalonil was used. Severity of late blight was significantly reduced in plots treated with fungicide $(P<0.05)$ relative to the unsprayed plots (Table 1$)$. No significant difference in disease suppression was observed between the DSS strategies and the calendar-based strategies on either category of cultivar resistance $(P<0.05)$ (Table 1$)$. However, in this experiment disease progress on Yukon Gold was limited by plant senescence, which accounted for the lack of significant difference in disease severity between untreated plots of Yukon Gold (susceptible) and untreated Kennebec (moderately resistant). For Yukon Gold, the DSS scheduled three chlorothalonil and two mefenoxam + chlorothalonil applications (Table 1). For Kennebec, the DSS strategy scheduled two sprays of chlorothalonil and two sprays of mefenoxam + chlorothalonil applications (two sprays). For the calendar-based schedule, both Yukon Gold and Kennebec received three sprays of chlorothalonil and three sprays of mefenoxam + chlorothalonil.

\section{DISCUSSION}

The purpose of this study was to evaluate the utility of the BlightPro DSS for in-season late blight management. To evaluate the recommendations of the DSS under an extended set of environmental conditions, simulation analysis was conducted using weather data from locations in six potato-producing states over 14 years, producing 768 environments. As expected, host resistance and weather influenced the number of fungicide applications recommended by the DSS.

In situations with favorable weather for late blight and when a susceptible cultivar is selected, the DSS recommended more fungicide applications than a weekly schedule but with improved disease suppression. On average, the DSS recommended fewer fungicide applications on moderately susceptible cultivars than did the calendar schedule. For moderately susceptible cultivars, the average resultant disease suppression for the DSS schedule was $97.7 \%$, compared with the disease suppression achieved by weekly applications on susceptible cultivars (baseline), which was slightly higher $(98.6 \%)$. Although this difference is not large (1.4\% disease compared with $2.3 \%$ disease), it is important because we have set a goal of achieving disease suppression at least as effective as a calendar-based approach on susceptible cultivars. The effect of moderate resistance translated to significant reduction in average number of fungicide applications recommended by the DSS (35\% reduction), with disease suppression equivalent to the baseline weekly schedule on susceptible cultivars. The results of this evaluation are consistent with previous studies that investigated the ability of the forecasting systems, Blitecast and Simcast, to manage late blight of potato (Fry et al. 1983; Shtienberg and Fry 1990; Spadafora et al. 1984).

The efficacy of individual sprays in late blight disease suppression varies depending on the timing of the application relative to the date of inoculation or infection (Shtienberg et al. 1989). However, fungicide use efficiency (E) may be used for overall comparison of fungicide-scheduling methods because it expresses an average figure for each spray's efficiency at suppressing disease
(Shtienberg and Fry 1990). The return (in percent disease control terms) of sprays scheduled according to the DSS-recommended strategy was higher than that for the calendar-based method for all categories of late blight resistance. In addition, fungicide use efficiency increased when fungicide use was combined with higher levels of late blight resistance of the cultivar.

Two scenarios for initial appearance of disease in each season were investigated in simulation analyses. First, the initial appearance of late blight was set to occur 6 days after the accumulation of 18 Blitecast severity values. Second, the initial appearance of late blight was a random date between accumulation of 18 Blitecast severity values and the end of the season (random inoculation date scenario); this scenario was included to represent the variability in initial late blight occurrence due to differences in inoculum source. We were interested to determine whether the DSS recommendations maintained their additional benefit over the calendar-based strategy under conditions where the disease might not appear from primary inoculum in the immediate area.

Under the scenario where late blight was initiated 6 days after 18 severity values had accumulated, the DSS demonstrated significant improvement in fungicide use efficiency relative to the calendarbased schedule for all resistance categories. As would be expected, fungicide use efficiency declined under the random inoculation date scenario, because initiation of fungicide schedules occurred independently of the appearance of the disease and, therefore, sprays prior to disease appearance did not always contribute to disease control. The DSS schedule was less efficient than the calendar-based schedule for the susceptible category but was more efficient for moderately susceptible and moderately resistant categories. This suggests the importance of identifying the appropriate time to begin spraying. If sprays are initiated earlier than necessary, this can reduce fungicide use efficiency. Mechanisms to further refine the identification of high-risk inoculation events could improve fungicide use efficiency.

It should be noted that, although the fungicide use efficiency for susceptible cultivars was reduced under the variable inoculation date scenario, disease suppression with the DSS schedule was significantly improved relative to the calendar-based approach. Due to the threat of potato tuber blight, potato producers in the Northeast region of the United States strive to prevent even low levels of disease in order to reduce the possibility of tuber blight infections. Therefore, even relatively small reductions in foliar disease severity would likely be preferred by growers. In the current study, tuber blight was not evaluated in field experiments or simulation analyses.

In field experiments, both DSS and calendar-based fungicide scheduling methods suppressed late blight effectively, with no significant differences in final disease levels. The scheduling methods differed in the number of sprays recommended. The DSS schedule was influenced by prevailing weather and cultivar resistance and resulted in fewer fungicide sprays on cultivars with moderate susceptibility or resistance to late blight, or when weather conditions were less favorable for late blight. In seasons with weather favorable for late blight, the DSS recommended the same number or more sprays on susceptible cultivars relative to the calendar-based scheduling method. These results were in agreement with results from the simulation analyses.

This DSS is the first to integrate real-time location-specific observed and forecast weather (National Digital Forecast Database) to drive these forecasting systems, as well as the LATEBLIGHT simulator (LB2004 version with fungicide submodels), to enable informed in-season decision making. A previous study by Raposo et al. (1993) determined that there was a benefit to incorporating forecast weather information into the disease forecasting systems. However, the improvement in disease management (reduction in AUDPC) realized depended on the accuracy of the weather forecast and ranged from $5 \%$ reduction in disease severity for weather forecasts available in 1993 to $10 \%$ reduction if perfect knowledge of future 
weather 1 and 2 days in advance was available (Raposo et al. 1993). Additionally, weather forecasts provided an increased benefit in environments that were less favorable for late blight development (Raposo et al. 1993). It should be noted that observed and forecast weather information were utilized to provide DSS-recommended schedules for field experiments but DSS-recommended schedules for simulation analyses were created using only historic records of observed weather. In the simulation experiments, actual weather forecasts were not available. Historic records of observed weather data could not be used to generate forecasts, because the historic observed weather data are equivalent to perfect knowledge of future weather and, therefore, do not include the inherent variability due to in accurate weather forecasts.

The model we used simulates the effects of environment and cultivar resistance on the development of $P$. infestans, and includes a submodel for the initial deposition of the fungicide chlorothalonil and its subsequent weathering, redistribution, loss, and efficiency. The models used for simulation of the disease have been developed and improved over the past four decades (Andrade-Piedra et al. 2005b; Bruhn and Fry 1981; Doster et al. 1990). The models predict disease development in small plots of potato, and all tests of predictions have been done in small plots. Evaluation of the model predictions have been compared with observed epidemics in small plots over 100 times, under diverse environmental conditions (Andrade-Piedra et al. 2005a,c; Shtienberg and Fry 1990). The models are useful tools to compare the effects of treatments applied in small plots (Shtienberg and Fry 1990). Thus, the model should be a good predictor of results from small field plots, and is appropriately used when a large number of field experiments is prohibitively expensive and time consuming (Raposo et al. 1993). It should be noted that limitations of small-plot field experiments will apply equally to the conclusions from simulation analyses.

In simulation analyses, disease suppression for DSS schedules with moderately susceptible cultivars did not meet our criterion of disease suppression at least equivalent to the average for a weekly schedule on a susceptible cultivar. The DSS forecasting systems have since been adjusted to improve disease suppression for the moderately susceptible category by modifying the critical thresholds (blight units and fungicide units) for this resistance category based on simulation results. Optimization of DSS forecasting systems is underway to maximize fungicide use efficiency for all categories of cultivar resistance while maintaining disease suppression.

The current system provides recommendations for variableinterval fungicide application. In certain production systems, there can be limited flexibility around application intervals; to accommodate these situations, we are currently investigating mechanisms to include variable dose rather than variable time of fungicide application.

The Simcast forecasting system was initially developed for late blight of potato but extension of the system is underway to enable its use for late blight of tomato. Preliminary field testing of the system for tomato late blight management has demonstrated that the DSS recommendations can be used successfully.

Future research will include the addition of existing forecasting tools for other important foliar diseases of potato and tomato, such as early blight.

Many DSSs have been developed for plant diseases, and experimental testing has demonstrated their ability to improve disease suppression and lower risk of crop damage; however, some of these systems have been used widely and others have not (Shtienberg 2013). In the case of intensive crops and disease systems, such as late blight of potato and tomato, farm managers attempt to minimize the risk of performing a false-negative action (not spraying when spraying was necessary) (Shtienberg 2013). As stated by Shtienberg, "The farmer's main concern is not only to minimize the average cost of the control strategy, but also to avoid extremely large variation" (Shtienberg 2013). The results from this study have demonstrated that the use of disease forecast-driven recommendations can deliver improved disease management and reduce variability in disease suppression relative to a 7-day calendar-based management strategy. A secondary benefit is that fungicide use can be reduced when conditions are not favorable for late blight, or when partially resistant varieties are grown. Furthermore, the risk calculations of managers of intensive crops are likely to change as a result of changing regulatory pressures and public perceptions of pesticide use, which will accelerate the adoption and use of DSSs in these crops (Shtienberg 2013).

Technologies such as DSSs are key components of the precision agriculture (smart farming) approach. The outputs of this DSS are meant to aid decisions by the grower or the consultant. Rather than replace farmer expertise and gut feeling, DSSs such as BlightPro can help users maximize the efficiency of their crop protection strategy by enabling well-informed decisions. The system is not intended to replace grower or consultant decisions.

\section{ACKNOWLEDGMENTS}

We thank S. P. McKay and R. M. Randolph for their assistance with field work, two Phytopathology reviewers for very helpful comments, and the Cornell Statistical Consulting Unit for assistance with statistical analyses. This research was supported by the Agriculture and Food Research Initiative Competitive Grants Program (grant number 2011-68004-30154) from the United States Department of Agriculture (USDA), by USDA RIPM, by the Empire State Potato Growers Association, and by the College of Agriculture and Life Sciences at Cornell University.

\section{LITERATURE CITED}

Andrade-Piedra, J., Forbes, G., Shtienberg, D., Grunwald, N., Chacon, M., Taipe, M., Hijmans, R., and Fry, W. 2005a. Qualification of a plant disease simulation model: Performance of the LATEBLIGHT model across a broad range of environments. Phytopathology 95:1412-1422.

Andrade-Piedra, J., Hijmans, R., Forbes, G., Fry, W., and Nelson, R. 2005b. Simulation of potato late blight in the Andes. I: Modification and parameterization of the LATEBLIGHT model. Phytopathology 95:1191-1199.

Andrade-Piedra, J., Hijmans, R., Juarez, H., Forbes, G., Shtienberg, D., and Fry, W. 2005c. Simulation of potato late blight in the Andes. II: Validation of the LATEBLIGHT model. Phytopathology 95:1200-1208.

Bruhn, J. A., and Fry, W. E. 1981. Analysis of potato late blight epidemiology by simulation modeling. Phytopathology 71:612-616.

Cooke, L. R., Schepers, H. T. A. M., Hermansen, A., Bain, R. A., Bradshaw, N. J., Ritchie, F., Shaw, D. S., Evenhuis, A., Kessel, G. J. T., Wander, J. G. N., Andersson, B., Hansen, J. G., Hannukkala, A., Naerstad, R., and Nielsen, B. J. 2011. Epidemiology and Integrated Control of Potato Late Blight in Europe. Potato Res. 54:183-222.

Danies, G., Small, I. M., Myers, K., Childers, R., and Fry, W. E. 2013. Phenotypic characterization of recent clonal lineages of Phytophthora infestans in the United States. Plant Dis. 97:873-881.

Doster, M. A., Milgroom, M. G., and Fry, W. E. 1990. Quantification of factors influencing potato late blight suppression and selection for metalaxyl resistance in Phytophthora infestans - A simulation approach. Phytopathology 80:1190-1198.

Doster, M. A., Sweigard, J. A., and Fry, W. E. 1989. The Influence of host resistance and climate on the initial appearance of foliar late blight of potato from infected seed tubers. Am. Potato J. 66:227-233.

Fry, W. E. 1977. Integrated control of potato late blight-Effects of polygenic resistance and techniques of timing fungicide applications. Phytopathology 67:415-420.

Fry, W. E., Apple, A. E., and Bruhn, J. A. 1983. Evaluation of potato late blight forecasts modified to incorporate host-resistance and fungicide weathering. Phytopathology 73:1054-1059.

Fry, W. E., and Goodwin, S. B. 1997. Re-emergence of potato and tomato late blight in the United States. Plant Dis. 81:1349-1357.

Fry, W. E., McGrath, M. T., Seaman, A., Zitter, T. A., McLeod, A., Danies, G., Small, I. M., Myers, K., Everts, K., Gevens, A. J., Gugino, B. K., Johnson, S. B., Judelson, H., Ristaino, J., Roberts, R., Secor, G., Seebold, K., Jr., Snover-Clift, K., Wyenandt, A., Gruenwald, N. J., and Smart, C. D. 2013. The 2009 late blight pandemic in the Eastern United States-Causes and results. Plant Dis. 97:296-306.

Gorry, G. A., and Morton, M. S. S. 1971. Framework for management information systems. Sloan Manage. Rev. 13:55-70.

Hardwick, N. V. 2006. Disease forecasting. Pages 239-267 in: The Epidemiology of Plant Diseases. B. M. Cooke, D. G. Jones, and B. Kaye, eds. Springer, the Netherlands. 
Haverkort, A. J., Boonekamp, P. M., Hutten, R., Jacobsen, E., Lotz, L. A. P., Kessel, G. J. T., Visser, R. G. F., and van der Vossen, E. A. G. 2008. Societal costs of late blight in potato and prospects of durable resistance through cisgenic modification. Potato Res. 51:47-57.

Hsu, J. C. 1996. Multiple Comparisons: Theory and Methods, 1st ed. Chapman \& Hall, London, New York. doi:10.1007/978-1-4899-7180-7

Hyre, R. A. 1954. Progress in forecasting late blight of potato and tomato. Plant Dis. Rep. 38:245-253.

Johnson, D., Cummings, T., Hamm, P., Rowe, R., Miller, J., Thornton, R., Pelter, G., and Sorensen, E. 1997. Potato late blight in the Columbia Basin: An economic analysis of the 1995 epidemic. Plant Dis. 81:103-106.

Jones, A. L., Lillevik, S. L., Fisher, P. D., and Stebbins, T. C. 1980. A microcomputer-based instrument to predict primary apple scab infection periods. Plant Dis. 64:69-72.

Knight, J. D. 1997. The role of decision support systems in integrated crop protection. Agric. Ecosyst. Environ. 64:157-163.

Krause, R. A., Massie, L. B., and Hyre, R. A. 1975. Blitecast-Computerized forecast of potato late blight. Plant Dis. Rep. 59:95-98.

Lees, A., Wattier, R., Shaw, D., Sullivan, L., Williams, N., and Cooke, D. 2006. Novel microsatellite markers for the analysis of Phytophthora infestans populations. Plant Pathol. 55:311-319.
Madden, L., Pennypacker, S. P., and MacNab, A. A. 1978. FAST, a forecast system for Alternaria solani on tomato. Phytopathology 68:1354-1358.

Raposo, R., Wilks, D. S., and Fry, W. E. 1993. Evaluation of potato late blight forecasts modified to include weather forecasts: A simulation analysis. Phytopathology 83:103-108.

Shtienberg, D. 2013. Will decision-support systems be widely used for the management of plant diseases? Annu. Rev. Phytopathol. 51:1-16.

Shtienberg, D., Doster, M. A., Pelletier, J. R., and Fry, W. E. 1989. Use of simulation models to develop a low-risk strategy to suppress early and late blight in potato foliage. Phytopathology 79:590-595.

Shtienberg, D., and Fry, W. E. 1990. Field and computer simulation evaluation of spray-scheduling methods for control of early and late blight of potato. Phytopathology 80:772-777.

Small, I. M., Joseph, L., and Fry, W. E. 2015. Development and implementation of the BlightPro decision support system for potato and tomato late blight management. Comput. Electron. Agric. 115:57-65.

Spadafora, V. J., Bruhn, J. A., and Fry, W. E. 1984. Influence of selected protectant fungicides and host resistance on simple and complex potato late blight forecasts. Phytopathology 74:519-523.

Wallin, J. R. 1962. Summary of recent progress in predicting late blight epidemics in the United States and Canada. Am. Potato J. 39:306-312. 